\title{
The Electromagnetic Wave Propagation on the Interface between Low and High Loss Dielectrics
}

\author{
Z. E. Eremenko \\ Institute for Radiophysics and Electronics of Ukrainian National Academy of Sciences of Ukraine, 12 Proskura Street, \\ Kharkov 61085, Ukraine \\ Correspondence should be addressed to Z. E. Eremenko, zoya.eremenko@gmail.com
}

Received 9 September 2010; Accepted 2 November 2010

Academic Editor: Mohindar S. Seehra

Copyright ( 2010 Z. E. Eremenko. This is an open access article distributed under the Creative Commons Attribution License, which permits unrestricted use, distribution, and reproduction in any medium, provided the original work is properly cited.

The wave propagation on closed curved interface (a rod or ball) between low and high loss dielectrics was studied and compared with well-known wave propagation problem on flat plane interface between low loss and high loss media. We have studied the propagation of a cylindrical wave along a round dielectric rod immersed into a high loss medium and a spherical wave forming by the oscillations in a dielectric ball immersed into a high loss medium as well. These waves have surface character similar to the wave known as the Zenneck surface wave. The distinctive characteristic of such cylindrical or spherical waves: the more loss in high loss medium the greater its surface character. We showed that the wave attenuation is essentially small at enough big dissipative loss in outer medium of dielectric structures.

\section{Introduction}

In the paper we study the wave propagation on the boundary of a high loss medium and a low loss dielectric flat plane, a round rod, and a ball. This wave has the surface character similar to the wave known as the Zenneck surface wave. The theoretical study of the surface wave started by Sommerfeld for electromagnetic wave propagation on a single metal wire [1], followed by Zenneck's theoretical description of electromagnetic wave propagation on a flat metal surface [2]. For a long time the possibility of its existence and experimental observation have been discussed in the literature [3-11]. This wave can propagate on the surface of low loss dielectric (in particular, open air) and high loss medium, such as the sea and ground. They considered that it can propagate with small attenuation. Earlier (in 3040 years of 20-th century) a number of scientists supposed that the Zenneck wave can be used for a long distance radio communication and that is why such a wave was under great consideration. The large-scale experiment was carried out in the Soviet Union (1934-1941), however, the efforts to excite this wave over the ground or sea failed [12].
The distinctive feature of the Zenneck wave is its strong attenuation and, therefore, in practice, it does not propagate along the surface. It can be explained as follows. First, the wave field penetrates into the ground and strongly attenuates in it. Second, because of big vertical field extension in the air the efficiency of excitation of the Zenneck wave by custom transmitting antenna is rather small. That is why at present the experiment observation of the Zenneck wave itself is not enough convincing and is in need of verification $[8,9]$.

We should mention that the wave characteristics propagated along the dielectric and high loss medium interface depend on complex permittivity of high loss medium and these characteristics can be under control changing the configuration and dielectric properties of the system where the wave is excited. It gives the opportunity to use such waves for complex permittivity measurement of medium, in particular, high loss liquid. It was studied theoretically and experimentally in our papers $[13,14]$.

In paper [13] we considered a dielectric ball placed into high loss liquid at millimeter wave band. So far as the surround liquid has rather small wave resistance, the ball has good resonant characteristics. It is well known that the ball has eigen "whispering gallery" oscillations [15]. If the outer 
medium of the ball is high loss liquid, we obtained that the $Q$-factor of such eigen oscillations in this resonator are of order of several decades and strong depend on complex permittivity of the ball and the surrounded liquid.

Also the dielectric rod surrounded by high loss liquid has been studied in [14]. It is well known that a dielectric rod has eigen waves as a dielectric waveguide. We showed the electromagnetic wave has strong attenuation along the surface normal. The wave velocity strongly depends on complex permittivity of the rod and the liquid. This feature we used to design differential complex permittivity measurement method at millimeter wave band. This method has enough high sensibility (relative measurement error is about $0.01 \%$ ) and allows to obtain rather small difference in dielectric properties of two high loss liquids, one if which is a reference liquid. Based on that method we have designed a millimeter wave dielectometer which is used to detect fabrication wines and fruit juices under the support by Science and Technology Center in Ukraine (project no.3870).

The goal of the present paper is the study of conditions and the determination of propagation parameters of the wave similar to the Zenneck wave along the curved surface between low loss dielectric and high loss medium. We also present the wave propagation results for flat plane surface where the Zenneck wave was originally theoretically predicted.

\section{The Wave on the Flat Plane Interface}

At first we consider the propagation ability of the wave that is similar to the wave discovered by Zenneck in 1907 [2], that is, the propagation of the surface wave along flat plane between two dielectrics one of them has high loss. We will solve the electrodynamics problem without external excitation sources (eigenvalue problem). Let us considered transverse-electric (TE) wave along $x$-axis on the interface between two dielectrics [16]. The half-space with $z>0$ is filled by the medium 1 with complex permittivity as $\varepsilon_{1}=$ $\varepsilon_{1}^{\prime}+i \varepsilon_{1}^{\prime \prime}$, and at $z<0$ there is medium 2 with complex permittivity as $\varepsilon_{2}=\varepsilon_{2}^{\prime}+i \varepsilon_{2}^{\prime \prime}$, where

$$
\varepsilon_{1}^{\prime}<\varepsilon_{2}^{\prime}, \quad \varepsilon_{1}^{\prime \prime} \ll \varepsilon_{2}^{\prime \prime}, \quad \frac{\varepsilon_{2}^{\prime \prime}}{\varepsilon_{2}^{\prime}}>1 .
$$

The time dependence we take in the present paper study as $\exp (-i \omega t)$. The electromagnetic field components are given as

$$
\begin{aligned}
& H_{1}=H_{0} \exp \left(i k_{x} x-k_{z 1} z\right), \quad z>0, \\
& H_{2}=H_{0} \exp \left(i k_{x} x+k_{z 2} z\right), \quad z<0,
\end{aligned}
$$

where $k_{z 1}=\sqrt{k_{x}^{2}-k^{2} \varepsilon_{1}}$ and $k_{z 2}=\sqrt{k_{x}^{2}-k^{2} \varepsilon_{2}}$ are transverse wave numbers. $k$ is wave number in vacuum, $k=\omega / c, \omega$ is round frequency, and $c$ is light velocity in vacuum. Using the continuity condition for $H_{y}$ and $E_{x}$ field components on the surface $z=0$, we can write the characteristic equation as follows [16]:

$$
-\frac{k_{z 1}}{\varepsilon_{1}}=\frac{k_{z 2}}{\varepsilon_{2}} .
$$

The radiation condition at infinity in medium 1 at $z>0$ has the view: $k_{z 1}^{\prime}>0$ and in medium 2 at $z<0: k_{z 2}^{\prime}>0$. It is necessary to say that (3) does not have any solution without loss at least in one of the considered media. The results of numerical calculation of characteristics equation (3) present in Figure 1 for the dependence of wave attenuation and phase velocity on the imaginary complex permittivity parts $\varepsilon_{2}^{\prime \prime}$. The algorithm to define the roots of a complex equation described in [17].

We can see that wave attenuation is increased at small values $\varepsilon_{2}^{\prime \prime}\left(0<\varepsilon_{2}^{\prime \prime}<\varepsilon_{2}^{\prime}\right)$, and phase velocity reduces. It corresponds to the decrease of electromagnetic field volume in media 1 and 2 . It can be confirmed by the dependence in Figures 2(a) and 2(b), where the real parts of transverse wave numbers $k_{z 1}^{\prime}$ and $k_{z 2}^{\prime}$ increase simultaneously in this case. At $\varepsilon_{2}^{\prime \prime} \approx \varepsilon_{2}^{\prime}$ there is a maximum for wave attenuation and $k_{z 1}^{\prime}$ values. At further increase of $\varepsilon_{2}^{\prime \prime}\left(\varepsilon_{2}^{\prime \prime}>\varepsilon_{2}^{\prime}\right)$ value $k_{z 1}^{\prime}$ is decreased but $k_{z 2}^{\prime}$ is increased and the wave attenuation is increased as well, that is, the field volume in medium 1 is increased and this field radiates stronger into this medium. At $k_{z 2}^{\prime}$ increasing the field drives into the interface on the high loss medium side. Here there is so-called pushing-out effect for the electromagnetic field in high loss medium [17-19].

It is necessary to note that the phase velocity of the wave is more than the light velocity in medium 1 (fast mode) for media with $\varepsilon_{2}^{\prime \prime}<100$. At $\varepsilon_{2}^{\prime \prime}>100$ the phase velocity of the wave is practically equal to the light velocity. In other words, this wave is similar to the wave that propagates along the metal wire [11]. However, the present study assumes that the medium are generally optically thick. For example, this is the reason why the wave's phase velocity at the surface of a metal approaches the speed of light in vacuum, as the field hardly penetrates the material. However, this is no longer true for thin metal wires or layers, in the optical range for example, where the index of refraction of metals may approach unity and field penetration may be significant.

\section{The Wave on the Cylindrical Interface}

Let us consider the electromagnetic wave propagation $H_{11}$ in a round cylinder rod of radius $a$ filled with small loss dielectric $\left(\varepsilon_{1}^{\prime} \gg \varepsilon_{1}^{\prime \prime}\right)$. Complex permittivity of media inside and outside of the rod are $\varepsilon_{1}=\varepsilon_{1}^{\prime}+i \varepsilon_{1}^{\prime \prime}$ and $\varepsilon_{2}=\varepsilon_{2}^{\prime}+$ $i \varepsilon_{2}^{\prime \prime}$, respectively. If $\varepsilon_{1}^{\prime}>\varepsilon_{2}^{\prime}$, than owing to the full internal reflection effect $[11,15]$ in the rod, there exist eigen cylinder waves of "whispering gallery" type. The field maximum of such waves concentrates inside of the rod nearby the rod surface and possesses fast attenuation in radial direction both inside and outside of the surface. Another situation arises when there is opposite inequality such as $\varepsilon_{1}^{\prime}<\varepsilon_{2}^{\prime}$. As it will be described below, the wave attenuation in the rod is getting smaller at the loss increase in outer medium. Such a cylinder wave can along the cylinder rod placed into high loss medium. As it was shown in [13], the wave characteristics strongly depend on dielectric properties of the outer medium and, then it is similar to the surface Zenneck wave, since it can be called cylindrical Zenneck wave. We assume that the relation between real and imaginary complex permittivity 


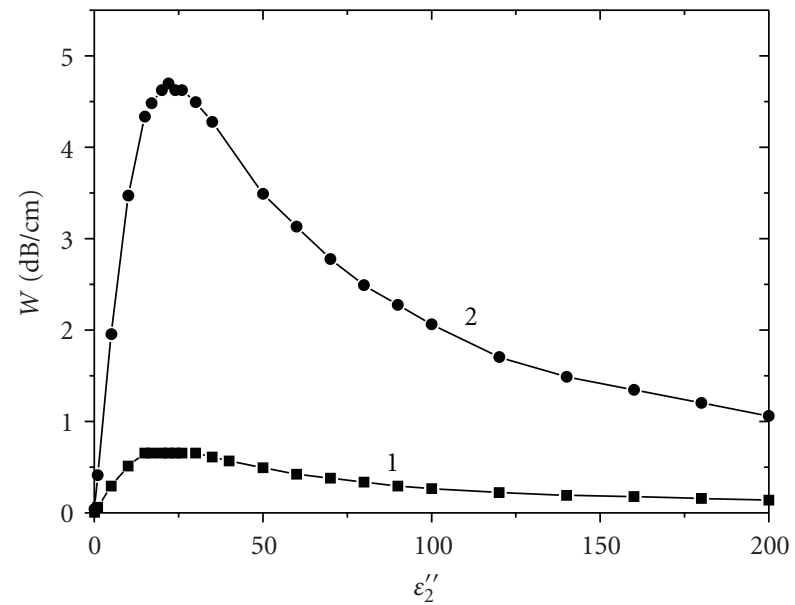

(a)

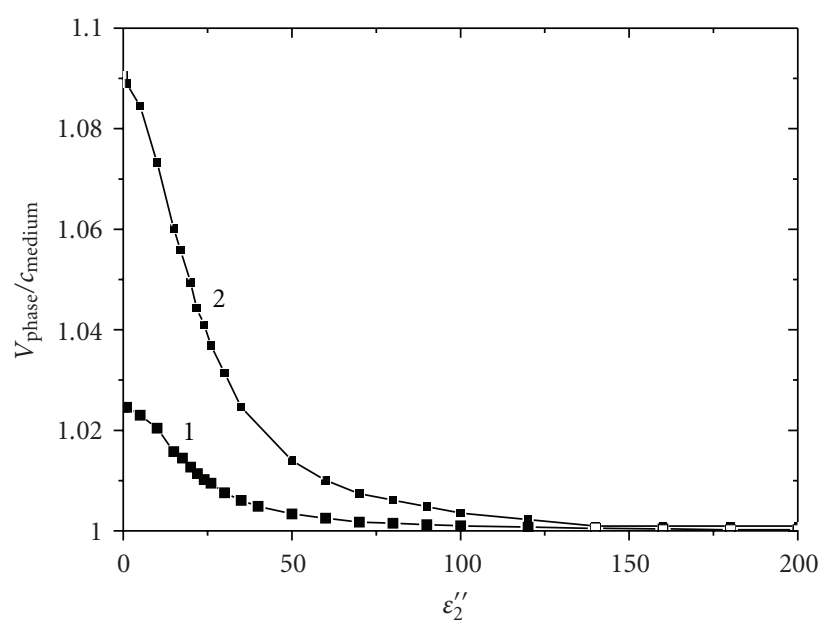

(b)

FIGURE 1: The wave attenuation $W$ (a) and normalized phase velocity to the light velocity in medium $1, V_{\text {phase }} / c_{\text {mediun }}$ (b) on imaginary complex permittivity part $\varepsilon_{2}^{\prime \prime}$ for the wave propagating along flat plane interface between low loss dielectrics and high loss media at frequency $31.82 \mathrm{GHz}, \varepsilon_{2}=20+i \varepsilon_{2}^{\prime \prime}$ Curves 1 are for $\varepsilon_{1}^{\prime}=1+i 0$ (open air), curve 2 are $\varepsilon_{1}^{\prime}=3.8+i 0.0001$ (quartz).

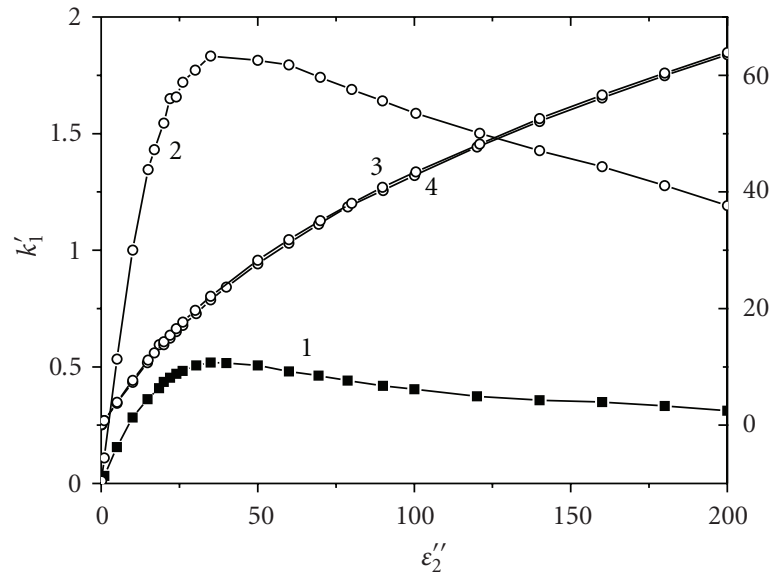

(a)

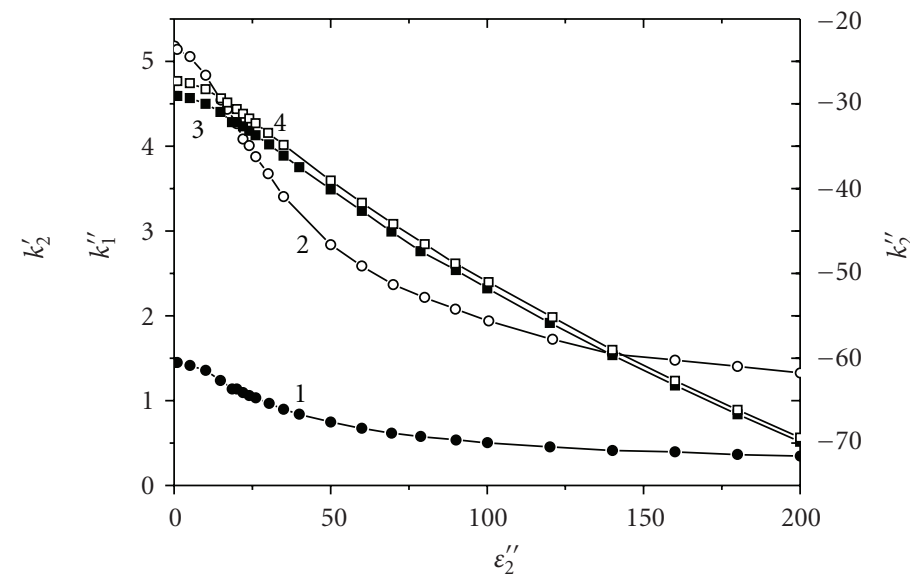

(b)

FIGURE 2: The dependence of real parts of wave numbers $k_{z 1}^{\prime}, k_{z 2}^{\prime}$ (a) and imaginary parts $k_{z 1}^{\prime \prime}, k_{z 2}^{\prime \prime}$ (b) on complex permittivity part $\varepsilon_{2}^{\prime \prime}$ for the wave propagating along flat plane interface between low loss dielectrics and high loss media. The operating frequency $31.82 \mathrm{GHz}$. The curves 1 and 3 are for $\varepsilon_{1}^{\prime}=1+i 0$ (open air), the curve 2 and 4 are for $\varepsilon_{1}^{\prime}=3.8+i 0.0001$ (quartz).

parts of the rod and outer medium are the same as (1). As known, waves in cylinder dielectric rod are hybrid [20], that is, they cannot be separated into electric and magnetic types. Thus, to obtain the characteristic equation for the rod they use electrical and magnetic Hertz components $[11,20]$. So, the electrical $(e)$ and magnetic $(m)$ Hertz vector projections $\Pi_{z}^{(e, m)}$ on $z$-axis in the rod and in the outer medium are as follows:

$$
\begin{aligned}
& \Pi_{z}^{(e)}=A J_{n}\left(g_{1} r\right) \cos (m \varphi) e^{i h z}, \quad r \leq a, \\
& \Pi_{z}^{(m)}=B J_{n}\left(g_{1} r\right) \sin (m \varphi) e^{i h z}, \\
& \Pi_{z}^{(e)}=C H_{n}^{(1)}\left(g_{2} r\right) \cos (m \varphi) e^{i h z}, \quad r>a, \\
& \Pi_{z}^{(m)}=D H_{n}^{(1)}\left(g_{2} r\right) \sin (m \varphi) e^{i h z},
\end{aligned}
$$

where $g_{1}=\sqrt{k^{2} \varepsilon_{1} \mu_{1}-h^{2}}, g_{2}=\sqrt{k^{2} \varepsilon_{2} \mu_{2}-h^{2}}, h$ is the propagation factor along $z$-axis, $A, B, C, D$ are field constants, $J_{n}(z), H_{n}^{(1)}(z)$ are the Bessel and Hankel functions of the first type, $n$ is the azimuthal index. The tangential field components are given as follows:

$$
\begin{aligned}
E_{\varphi} & =\frac{\partial^{2} \Pi_{z}^{e}}{\partial \varphi \partial z}-i k \mu \frac{\partial \Pi_{z}^{m}}{\partial r}, & H_{\varphi} & =\frac{\partial^{2} \Pi_{z}^{m}}{\partial \varphi \partial z}+i k \varepsilon \frac{\partial \Pi_{z}^{e}}{\partial r}, \\
E_{z} & =\left(\frac{\partial^{2}}{\partial z}+k^{2} \varepsilon \mu\right) \Pi_{z}^{e}, & H_{z} & =\left(\frac{\partial^{2}}{\partial z}+k^{2} \varepsilon \mu\right) \Pi_{z}^{m} .
\end{aligned}
$$

Then, the relations for electric and magnetic field components have the following view in the dielectric rod 


$$
\begin{gathered}
E_{\phi}=-i\left[\frac{n h}{r} A J_{n}\left(g_{1} r\right)+k g_{1} B J_{n}^{\prime}\left(g_{1} r\right)\right] \sin (n \phi) e^{i h z} \\
H_{\phi}=i\left[k g_{1} \varepsilon_{1} A J_{n}^{\prime}\left(g_{1} r\right)+\frac{n h}{r} B J_{n}\left(g_{1} r\right)\right] \cos (n \phi) e^{i h z} \\
E_{z}=g_{1}^{2} A J_{n}\left(g_{1} r\right) \cos (n \phi) e^{i h z} \\
H_{z}=g_{1}^{2} B J_{n}\left(g_{1} r\right) \sin (n \phi) e^{i h z}
\end{gathered}
$$

and in the outer medium

$$
\begin{gathered}
E_{\phi}=-i\left[\frac{n h}{r} C H_{n}^{(1)}\left(g_{2} r\right)+k g_{2} D H_{n}^{(1)^{\prime}}\left(g_{2} r\right)\right] \sin (n \phi) e^{i h z}, \\
H_{\phi}=i\left[k g_{2} \varepsilon_{2} C H_{n}^{(1)^{\prime}}\left(g_{2} r\right)+\frac{n h}{r} D H_{n}^{(1)}\left(g_{2} r\right)\right] \cos (n \phi) e^{i h z} \\
E_{z}=g_{2}^{2} C H_{n}^{(1)}\left(g_{2} r\right) \cos (n \phi) e^{i h z} \\
H_{z}=g_{2}^{2} D H_{n}^{(1)}\left(g_{2} r\right) \sin (n \phi) e^{i h z}
\end{gathered}
$$

where the prime denotes the derivative on argument. Satisfying boundary conditions on the rod surface $(r=a)$, we obtain the system of linear equations relatively to the parameter $h$

$$
a_{i j}(h)=0, \quad i, j=1 ; 2 ; 3 ; 4
$$

where

$$
\begin{aligned}
& a_{11}=\frac{h n J_{n}\left(g_{1} a\right)}{a}, \quad a_{12}=k g_{1} J_{n}^{\prime}\left(g_{1} a\right), \\
& a_{13}=-\frac{h n H_{n}^{(1)}\left(g_{2} a\right)}{a}, \quad a_{14}=-k g_{2} H_{n}^{(1)^{\prime}}\left(g_{1} a\right), \\
& a_{21}=k g_{1} \varepsilon_{1} J_{n}^{\prime}\left(g_{1} a\right), \quad a_{22}=\frac{h n J_{n}\left(g_{1} a\right)}{a}, \\
& a_{23}=-k g_{2} \varepsilon_{2} H_{n}^{(1)^{\prime}}\left(g_{2} a\right), \quad a_{24}=-\frac{h n H_{n}^{(1)}\left(g_{2} a\right)}{a}, \\
& a_{31}=a_{42}=g_{1}^{2} J_{n}\left(g_{1} a\right), \quad a_{33}=a_{44}=-g_{2}^{2} H_{n}^{(1)}\left(g_{2} a\right) \text {. }
\end{aligned}
$$

If the determinant of this system is equal to zero we can write the characteristic equation for the determination of the propagation coefficient $h$. This characteristic equation is as follows:

$$
\begin{aligned}
& \left(\frac{J_{1}^{\prime}\left(g_{1} a\right)}{g_{1} a J_{1}\left(g_{1} a\right)}-\frac{H_{1}^{(1)^{\prime}}\left(g_{2} a\right)}{g_{2} a H_{1}^{(1)}\left(g_{2} a\right)}\right) \\
& \quad \times\left(\frac{\varepsilon_{1} J_{1}^{\prime}\left(g_{1} a\right)}{g_{1} a J_{1}\left(g_{1} a\right)}-\frac{\varepsilon_{2} H_{1}^{(1)^{\prime}}\left(g_{2} a\right)}{g_{2} a H_{1}^{(1)}\left(g_{2} a\right)}\right)=\frac{h^{2}\left(g_{2}^{2}-g_{1}^{2}\right)^{2}}{k^{2} g_{1}^{4} g_{2}^{4} a^{4}} .
\end{aligned}
$$

Let us find the infinity conditions for the waves propagated along the considered rod. The solution of the wave equation [20]

$$
\Delta \psi+k^{2} \psi=0
$$

was taken as the Hankel function of the first type for outer medium [11]. So, this function can be written as follows at big arguments $($ at $R \rightarrow \infty)$ [21]

$$
\psi=H_{n}^{(1)}\left(g_{2} R\right)=\sqrt{\frac{2}{\pi g_{2} R}} \exp \left[i\left(g_{2} R-\frac{n \pi}{2}-\frac{\pi}{4}\right)\right] .
$$

Then, the wave attenuation at infinity [20] for the cylinder wave has the view

$$
\begin{aligned}
\lim _{R \rightarrow \infty} R^{3 / 2} \cdot\left(\frac{\partial \psi}{\partial R}-i g_{2} \psi\right) \\
=-\sqrt{\frac{1}{2 \pi g_{2}}} \exp \left[i\left(g_{2}^{\prime} R-\frac{n \pi}{2}-\frac{\pi}{4}\right)-g_{2}^{\prime \prime} R\right] .
\end{aligned}
$$

Therefore, in order to this limit tended to zero at infinity it is necessary to have imaginary part like this $g_{2}^{\prime \prime}>0$.

We can find wave parameters such as attenuation coefficient, phase velocity, and also electric and magnetic field components distribution of the cylinder wave in the rod and outer medium using numerical study of (4). In Figure 3 we present the numerical results for the attenuation and phase velocity for the cylinder wave propagation in the rod. Take into account the comparison with experiment, described in our paper [14], we took the rod material as quartz $\left(\varepsilon_{1}=3.8+i 0.0001\right)$ with diameter of $0.5 \mathrm{~cm}$, the operating frequency was taken as $31.82 \mathrm{GHz}$. Figure 3 contains experimental data points that were presented in [14] for comparison. These experimental data points correspond to different concentration of water-ethanol solutions that has different both real and imaginary parts of complex permittivity values $[22,23]$. The curves in Figure 3 were calculated for definite values of real part of outer medium complex permittivity $\left(\varepsilon_{2}^{\prime}=10\right.$ (curve 1 ) and $\varepsilon_{2}^{\prime}=20$ (curve 2)). Thus, the mentioned above experimental data points can coincide only with those numerical data which correspond to the data with definite concentration of waterethanol solutions.

As it is shown in Figure 3 the attenuation in outer medium rapidly decreases at the imaginary part of complex permittivity $\varepsilon_{2}^{\prime \prime}$ increase. It can be explained as follows. At the increase of wave attenuation in the outer medium the cylinder wave is concentrated in the rod (see Figure 4). For big values of the imaginary complex permittivity part $\left(\varepsilon_{2}^{\prime \prime} \gg 1\right)$ attenuation tends to zero and electrodynamics properties of the rod correspond to round metal waveguide in which the cylinder wave propagates with small dissipation. At small values of imaginary complex permittivity part $\left(\varepsilon_{2}^{\prime \prime} \rightarrow 0\right)$, as follows from Figure 3 , the wave is not concentrated and it propagates with small dissipation in outer medium. The phase velocity has minimum at $\varepsilon_{2}^{\prime \prime} \approx \varepsilon_{2}^{\prime}$. It can be explained as follows. At $0<\varepsilon_{2}^{\prime \prime}<\varepsilon_{2}^{\prime}$ the wave attenuation increases in outer medium and phase velocity reduces, at further increase of imaginary complex permittivity part $\varepsilon_{2}^{\prime \prime}$ the field volume in outer medium reduces (Figure 4) and the wave is concentrated in the rod with small dissipation. This leads to the phase velocity increase. 


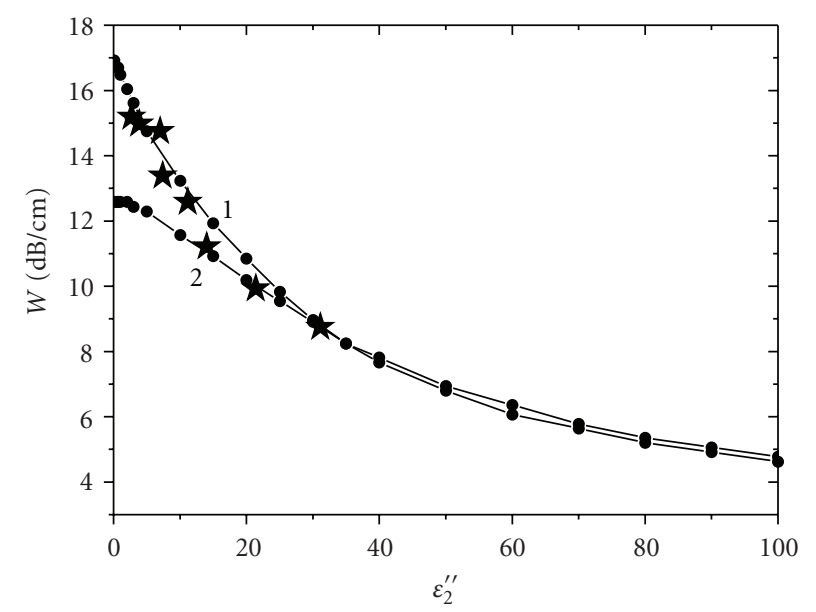

(a)

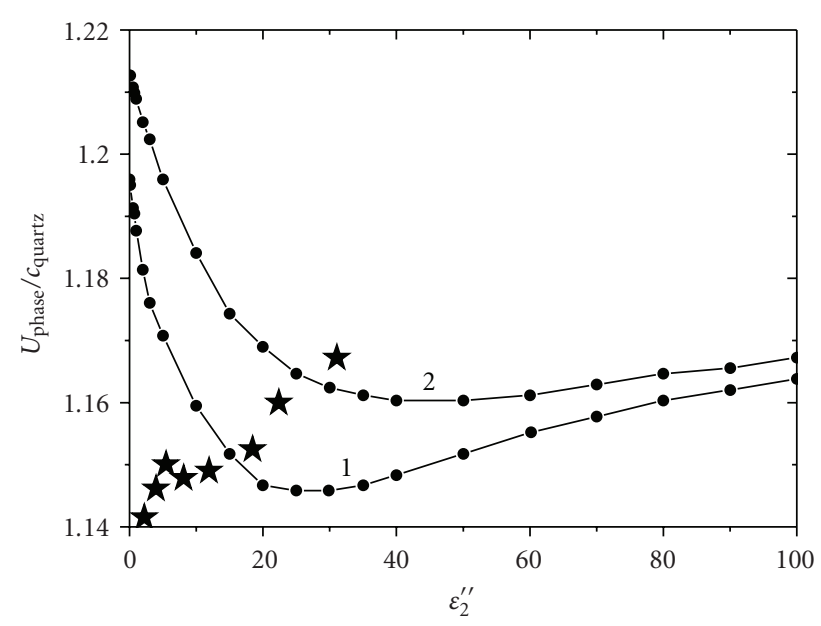

(b)

FIGURE 3: The dependence of the attenuation coefficient (a) and the normalized phase velocity $V_{\text {phase }} / c_{\text {quartz }}$ (b) to the light velocity in the quartz $\operatorname{rod}\left(\varepsilon_{1}=3.8+i 0.0001\right)$ for the wave in cylinder round rod. Complex permittivity of the outer medium is $\varepsilon_{2}^{\prime}=10$ (curve 1$)$ and $\varepsilon_{2}^{\prime}=20$ (curve 2). The operating frequency is $31.82 \mathrm{GHz}$. The stars denote experimental data points from [14] (the outer medium was taken as water-ethanol solutions at $23^{\circ} \mathrm{C}$ from $10 \%$ to $70 \%$ ethanol in water $[22,23]$ ).

At big values of imaginary complex permittivity part $\varepsilon_{2}^{\prime \prime}$ of outer medium the wave attenuation is rapidly reduces. At that the field outside of the rod is concentrated at the surface (Figure 4). In another words, it presents so-called pushingout effect for electromagnetic field in the high loss medium described in our papers [17-19].

In Figure 4 it is shown that the distribution of field component $H_{z}$ in the rod and in outer medium for the wave $H_{11}$ type. At that the field penetrates long after of rod border at rather small values of real and imaginary complex permittivity parts $\varepsilon_{2}$ : the relative depth of field penetration is $d / a \gg 1$, where $d$ is the distance out from the rod surface in radial direction. At the increase of the attenuation in outer medium the value $d / a$ reduces and the field is concentrated nearby the rod surface. The field is similar to the surface wave field at great dissipation in outer medium $\left(\varepsilon_{2}^{\prime \prime} \geq 10\right)$. At that it rapidly drops in radial direction inside of the rod and does not radiate into low loss dielectric rod medium (because of closed curved rod surface) in contrast to the Zenneck wave (at the flat plane interface).

It is necessary to note that the cylinder wave in the rod surrounded by high loss medium is similar to the wave which propagates in tunnels with loss borders. Such waves studied in the literature $[11,20,24]$ in order to study the condition of reliable radio communication in mines and other similar structures. However, out of the consideration in the mentioned above the literature there is a question about the influence of outer medium dielectric properties on the wave propagation. Finally, along with $H_{11}$ wave type in the dielectric rod a set of hybrid waves of $E H_{\mathrm{nm}}$ and $H E_{\mathrm{nm}}$ and azimuthal symmetric waves of $\mathrm{TE}_{01}$ and $\mathrm{TM}_{01}$ types can be excited as well.

\section{The Wave on the Spherical Interface}

Earlier in [13] we studied the electromagnetic oscillations in a dielectric ball placed in high loss liquid with great

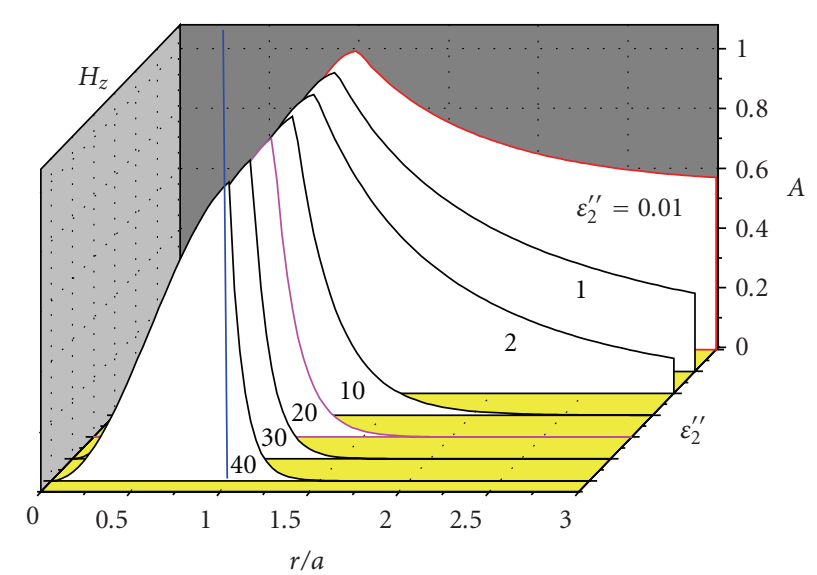

FIGURE 4: The normalized distribution of the $H_{z}$ field component for the rod in high loss medium. The relative distance $r / a$ in radial direction is across, the solid vertical line is the rod border, $\varepsilon_{2}^{\prime}=20$. The numbers denote the values of imaginary CP part $\varepsilon_{2}^{\prime \prime}=0.01 ; 1 ; 2 ; 10 ; 20 ; 30 ; 40$.

values of complex permittivity parts $\left(\varepsilon_{2}^{\prime}, \varepsilon_{2}^{\prime \prime} \gg 1\right)$. Since the ratio of wave impedance $\zeta=\sqrt{\left|\varepsilon_{1} / \varepsilon_{2}\right|}$ is small the ball possesses rather good resonance properties. These exist electromagnetic oscillations formed by spherical wave. Such a wave we can call as spherical Zenneck wave that is similar to the Zenneck wave on flat plane interface. At that the field of the spherical wave is concentrated nearby the inner spherical surface of the ball. The oscillation $Q$-factor can be enough high and strongly depends on complex permittivity of outer liquid surrounded the ball. As it will be described below the attenuation of the spherical wave as well as the attenuation of the cylinder wave in the dielectric rod sufficiently reduces at the increase of dissipative loss in outer medium and resonator $Q$-factor is increased, accordingly.

Let us consider the spherical wave in high loss medium. In order to detect such a wave we use a dielectric ball in 
high loss medium where the eigen oscillations formed by spherical waves are excited. The complex permittivity of a ball and an outer medium are $\varepsilon_{1}$ and $\varepsilon_{2}$, respectively. We consider a quasioptical case, when the ball radius is $a \gg \lambda$ ( $\lambda$ is an operating wave length). The characteristic equations for eigen oscillations in the ball are presented earlier in [13].

The Maxwell equation solutions spitted into the sum of electric (TM) transverse-magnetic) and magnetic (TE transverse-electric) types due to the assumptions that our media are isotropic and the system has spherical symmetry. Then, all electromagnetic field components can be expressed via scalar functions $U$ and $V[11,20]$. At that, these functions have to satisfy the following equation:

$$
\frac{\partial^{2} \Psi}{\partial r^{2}}+\frac{1}{r^{2} \sin \theta}\left[\frac{\partial}{\partial \theta}\left(\sin \theta \frac{\partial \Psi}{\partial \theta}\right)+\frac{1}{\sin \theta} \frac{\partial^{2} \Psi}{\partial \phi^{2}}\right] \Psi+k_{0}^{2} \varepsilon \mu \Psi=0,
$$

where $\Psi$ denotes $U$ or $V$. It is necessary to note though the functions $U$ and $V$ are defined by the same equation they are two different functions described the electric and magnetic fields. So, $U$ and $V$ define the electromagnetic fields of TM $\left(E_{r} \neq 0, H_{r}=0\right)$ and $\mathrm{TE}\left(E_{r}=0, H_{r} \neq 0\right)$ oscillations, respectively. The electromagnetic fields components using these functions we can write as follows for TM oscillations

$$
\begin{gathered}
E_{\theta}=\frac{1}{r} \frac{\partial^{2} U}{\partial \theta \partial r}, \quad E_{\phi}=\frac{1}{r \sin \theta} \frac{\partial^{2} U}{\partial \phi \partial r}, \quad E_{r}=\frac{\partial^{2} U}{\partial r^{2}}+k_{0}^{2} \varepsilon \mu U, \\
H_{\theta}=-\frac{i k_{0} \varepsilon}{r \sin \theta} \frac{\partial U}{\partial \phi}, \quad H_{\phi}=\frac{i k_{0} \varepsilon}{r} \frac{\partial U}{\partial \theta}, \quad H_{r}=0
\end{gathered}
$$

and for TE oscillations

$$
\begin{gathered}
H_{\theta}=\frac{1}{r} \frac{\partial^{2} V}{\partial \theta \partial r}, \quad H_{\phi}=\frac{1}{r \sin \theta} \frac{\partial^{2} V}{\partial \phi \partial r}, \quad H_{r}=\frac{\partial^{2} V}{\partial r^{2}}+k_{0}^{2} \varepsilon \mu V, \\
E_{\theta}=\frac{i k_{0} \mu}{r \sin \theta} \frac{\partial V}{\partial \phi}, \quad E_{\phi}=-\frac{i k_{0} \mu}{r} \frac{\partial V}{\partial \theta}, \quad E_{r}=0 .
\end{gathered}
$$

The Maxwell equation solution for the inside ball area $\left(0<r \leq r_{1}\right)$ for TM oscillations type we can write as follows:

$$
\Psi_{1}=C_{1} j_{n}\left(k_{0} \sqrt{\varepsilon_{1}} r\right) \chi(\theta, \phi) \exp (-i \omega t)
$$

and for the outer ball area $\left(r_{1}<r\right)$ we have

$$
\Psi_{2}=C_{2} y_{n}\left(k_{0} \sqrt{\varepsilon_{2}} r\right) \chi(\theta, \phi) \exp (-i \omega t),
$$

where $j_{n}(x)=\sqrt{\pi x / 2} J_{n+1 / 2}(x), y_{n}(x)=\sqrt{\pi x / 2} H_{n+1 / 2}^{(1)}(x)$, $\chi(\theta, \phi)=\chi_{\mathrm{TM}}(\theta, \phi)=P_{n}^{m}(\cos \theta) \cos m \phi ; J_{n+1 / 2}(x)$ and $H_{n+1 / 2}^{(1)}(x)$ are Bessel and Hankel function of the first order of half-integer indexes. It is necessary to note that functions $j_{n}(x)$ and $y_{n}(x)$ are not spherical ones defined in [20] due to factor $\sqrt{\pi x / 2}$. The values $\varepsilon_{1}$ and $\varepsilon_{2}=1$ are CP of the ball and outer medium respectively, $\mu_{1}=\mu_{2}=1 ; P_{n}^{m}(\cos \theta)$ is associated Legendre function of $n$ order and $m$ degree with argument $\cos \theta ; C_{j}$ are field coefficients $(j=1,2)$.
The solution for TE oscillations type differs by another coefficients (instead of $C_{j} \rightarrow D_{j}$ and $\chi(\theta, \phi)=\chi_{\mathrm{TE}}(\theta, \phi)=$ $\left.P_{n}^{m}(\cos \theta) \sin m \phi\right)$. We consider that $n$ is polar and $m$ is azimuth indexes of eigen oscillations in the ball, $l$ is radial index along the radial coordinate.

To satisfy the boundary conditions for the considered system it is enough the equality of tangential intensity components of electric and magnetic fields on the ball surface $\left(E_{\theta_{1}}=E_{\theta_{2}}, H_{\theta_{1}}=H_{\theta_{2}}\right)$. As a result we obtain an algebraic system equation for $C_{j}$ coefficients. Equating the determinant of this system to zero we have a characteristic equation to determine complex eigen frequencies of the dielectric ball in high loss medium. These equations have the following view for TM oscillations

$$
\sqrt{\varepsilon_{1}} j_{n}\left(k_{0} \sqrt{\varepsilon_{1}} a\right) y_{n}^{\prime}\left(k_{0} a_{1}\right)=\sqrt{\varepsilon_{2}} j_{n}^{\prime}\left(k_{0} \sqrt{\varepsilon_{1}} a\right) y_{n}\left(k_{0} a\right)
$$

and for TE type

$$
\sqrt{\varepsilon_{2}} j_{n}\left(k_{0} \sqrt{\varepsilon_{1}} a\right) y_{n}^{\prime}\left(k_{0} a\right)=\sqrt{\varepsilon_{1}} j_{n}^{\prime}\left(k_{0} \sqrt{\varepsilon_{1}} a\right) y_{n}\left(k_{0} a\right) .
$$

Let us find the infinity conditions for the waves propagated into the medium surrounded the ball. The solution $\psi$ of wave equation (12) in the outer medium for the open ball we take as follows [11]

$$
\psi=\sqrt{\frac{\pi g_{2}}{2 R}} H_{n+1 / 2}^{(1)}(g R) .
$$

The asymptote of Hankel function at big arguments is given as (13) [21]. The radiation condition for the spherical wave can be written as follows

$$
\lim _{R \rightarrow \infty} R^{2}\left(\frac{\partial \psi}{\partial R}-i g_{2} \psi\right)=-\exp \left[i\left(g_{2}^{\prime} R-\frac{n \pi}{2}-\frac{\pi}{4}\right)-g_{2}^{\prime \prime} R\right] .
$$

Then, in order to this limit tends to zero at infinity it is necessary to have imaginary part of number $g_{2}^{\prime \prime}>0$.

Numerical calculations of characteristic equation (9) for $\mathrm{TE}_{20 \mathrm{ml}}$ type of oscillations we present in Figure 5, where there is the dependence of $Q$-factor and resonance frequency shift on imaginary complex permittivity part of high loss outer medium. It is necessary to mention that the similar results are obtained for $\mathrm{TM}_{20 \mathrm{ml}}$ in [13].

As shown in Figure 5(a), Q-factor of the ball oscillations is sharply getting higher at loss increase in outer medium. Within limit it reaches the $Q$-factor value of oscillations in spherical resonator with metal borders. The dissipation in outer medium has an impact on eigen ball oscillation frequency. At small loss $\left(\varepsilon_{2}^{\prime \prime} \approx \varepsilon_{2}^{\prime}\right)$ the dependence of the frequency shift has an extremum similar to the flat plane interface case (Figure 1(a)) and the cylinder rod (Figure 3(b)).

The dependence of electromagnetic field distribution inside and outside the ball on dissipation in outer high loss medium presents in Figure 6. At small values $\varepsilon_{2}^{\prime \prime}$ the field is weakly confined by the ball and the wave propagates far out of the ball border. At the increase of $\varepsilon_{2}^{\prime \prime}$ the field is more and more concentrated nearby the ball interface and at great values of $\varepsilon_{2}^{\prime \prime}$ it has the characteristic of a surface wave similar to the Zenneck one. But this spherical wave is not radiated inside the low loss dielectric for flat plane interface described above. 


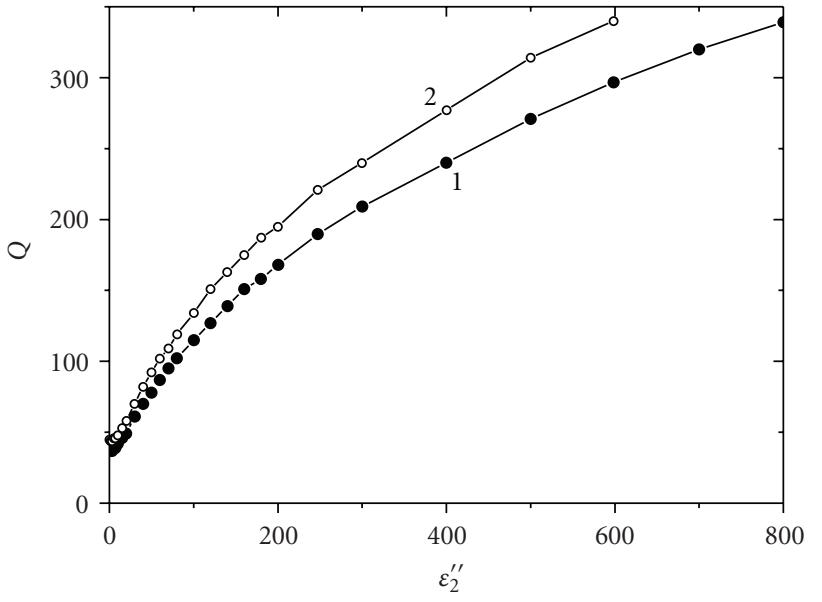

(a)

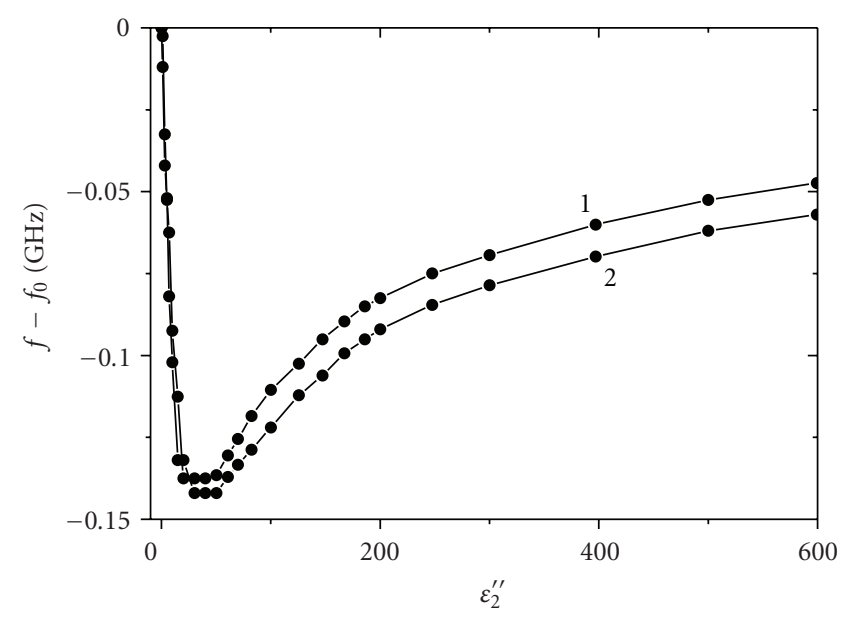

(b)

FIGURE 5: The dependence of $Q$-factor of the ball in high loss medium $Q$ (a) and resonance frequency shift $f$ (b) with respect to the frequency $f_{0}$ on imaginary CP part of high loss outer medium $\varepsilon_{2}^{\prime \prime}\left(f_{0}=31.82 \mathrm{GHz}\right.$ is the operating resonance frequency at $\left.\varepsilon_{2}^{\prime \prime}=0\right)$. The diameter of the ball is $2 a=5.56 \mathrm{~cm}$. Polar oscillation index is $n=20$ and radial index is $l=1$ (the curve 1 ) $l=2$ (the curve 2), $\varepsilon_{2}^{\prime}=20$. The stars denote experimental data points from [13] (the outer medium was taken as water-ethanol solutions at $23^{\circ} \mathrm{C}$ from $10 \%$ to $40 \%$ ethanol in water $[22])$.

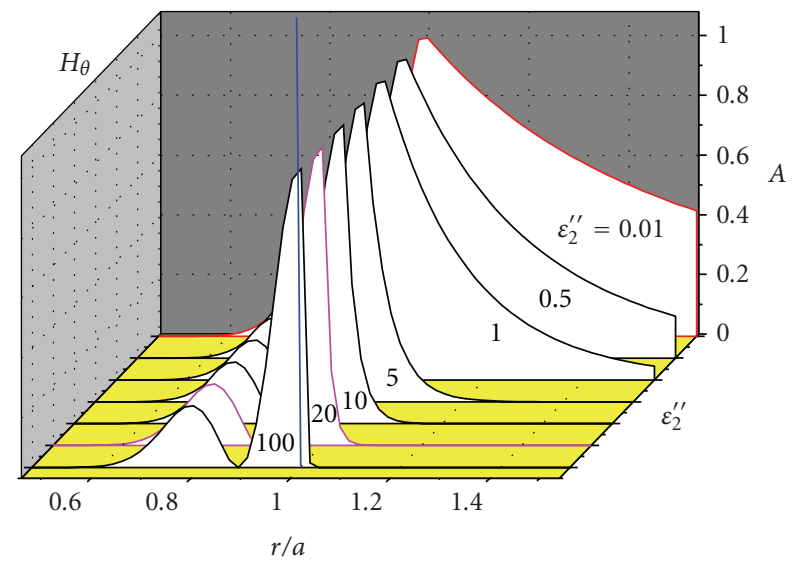

FIgURE 6: Normalized distribution of electromagnetic field component $H_{\theta}$ for the dielectric ball in high loss medium. The relative radial distance $r / a$ is along the horizontal axis. The ball border is a vertical solid line, $\varepsilon_{2}^{\prime}=20$. The numbers denote values $\varepsilon_{2}^{\prime \prime}=$ $0.01 ; 1 ; 5 ; 10 ; 20 ; 100$.

\section{Conclusion}

We studied the wave propagation along flat plane and curved interface between two dielectrics: the first one has small dielectric loss and the second one has high dielectric loss at millimeter wave band. The considered structures with curved interface were the round rod as a waveguide and the ball as a resonator. As a result we can say that the character of wave propagation along the dielectric-high loss medium interface is similar to the surface Zenneck wave characteristics by high loss media side.

The common features for the considered structures are as follows. First, at great values of imaginary complex permittivity part of high loss medium we have rather small attenuation for flat plane dielectric interface, the waveguide structure (the rod) or high $Q$-factor for the resonator structure (the ball). And there exists a pushing-out effect for the electromagnetic field in a high loss medium. The phase velocity of the waves for all these structures is a bit more than the light velocity in the dielectric medium. Thus, the considered waves are fast waves. It is an additional proof that these waves possess the Zenneck wave property. Another common feature of the considered structures is as follows. As the equality of real and imaginary complex permittivity part of high loss media there exist an extremum in wave propagation dependences. This point results the wave propagation character change from the wave attenuation from increase to decrease.

The main distinction between the Zenneck wave itself and the waves in studied structures with close curved interface is as follows. At great value of imaginary complex permittivity part of high loss medium the field volume in low loss dielectric increases and the wave strongly radiates out of the flat plane interface and, therefore, we cannot consider such a wave as a surface wave, in general. But for closed curved interface structures the field concentration along the interface both for low loss dielectric and for high loss medium is observed simultaneously.

Thus, the surface cylinder or spherical waves propagate along the closed cylinder or spherical interface, respectively, between the low loss dielectric and the high loss dielectric. This wave field is concentrated nearby the curved interface much more than the wave field on the flat plane interface. The distinctive feature of cylinder and spherical wave is the more dissipation in high loss medium the more field concentration nearby the curved interface. The characteristics of studied waves strongly depend on dielectric properties of 
high loss medium. It gives the opportunity to use such waves for complex permittivity measurements of high loss media $[14,17,18]$.

\section{Acknowledgment}

This work was partially supported by the Science and Technology Center of Ukraine (STCU), project no. 3870.

\section{References}

[1] A. Sommerfeld, "Uber die Fortpanzung elektrodynamischer Wellen langs eines Drahtes," Annalender Physik und Chemie, vol. 303, no. 2, pp. 233-290, 1899.

[2] J. Zenneck, "Uber die Fortpanzung ebener elektromagnetischer Wellen langs einer ebenen Leiterache und ihre Beziehung zur drahtlosen Telegraphie," Annalen der Physik, vol. 328, no. 10, pp. 846-866, 1907.

[3] A. A. Oliner, "Historical perspectives on microwave field," IEEE Transactions on Microwave Theory and Techniques, vol. 32, no. 9, pp. 1022-1045, 1984.

[4] A. Bano, Dipole Radiation in the Presence of a Conducting HalfSpace, Pergamon, Oxford, UK, 1966.

[5] A. Sommerfeld, Partial Differential Equations in Physics, Academic Press, New York, NY, USA, 1964.

[6] V. A. Foc, Electromagnetic Diffraction and Propagation Problems, Pergamon, Oxford, UK, 1965.

[7] J. R. Wait, "The ancient and modern history of EM groundwave propagation," IEEE Antennas and Propagation Magazine, vol. 40, no. 5, pp. 7-24, 1998.

[8] V. I. Baybakov, V. N. Dazhko, and Y. V. Kistovich, "Experimental detection of surface electromagnetic Zenneck waves," Uspekhi Fizicheskikh Nauk, vol. 157, no. 4, p. 722, 1989.

[9] A. V. Kukushkin, "On the existence and physical meaning of the Zenneck wave," Uspekhi Fizicheskikh Nauk, vol. 52, no. 7, pp. 755-756, 2009.

[10] M. Gong, T. I. Jeon, and D. Grischkowsky, "THz surface wave collapse on coated metal surfaces," Optics Express, vol. 17, no. 19, pp. 17088-17101, 2009.

[11] L. A. Vainstein, Electromagnetic Waves, Radio i Svjaz, Moscow, Russia, 1988.

[12] L. A. Mandelshtam and N. D. Papalexi, "Interenference methods of radiowave propagation study," in Sbornik: "Newrest Study of Radiowave Propagation", pp. 10-50, Gostexizdat, Moscow, Russia, 1945.

[13] Z. E. Eremenko and E. M. Ganapolskii, "Resonant spherical hole in a high loss liquid at millimeter wavelengths," IEEE Transactions on Microwave Theory and Techniques, vol. 54, no. 5, pp. 2243-2247, 2006.

[14] E. M. Ganapolskii, Z. E. Eremenko, and V. N. Skresanov, "A millimeter wave dielectrometer for high loss liquids based on the Zenneck wave," Measurement Science and Technology, vol. 20, no. 5, Article ID 055701, 8 pages, 2009.

[15] A. N. Oraevsky, "Whispering-gallery waves," Quantum Electronics, vol. 32, no. 5, pp. 377-400, 2002.

[16] L. Landau and E. Lifshitz, Electrodynamics of Continuous Media, Pergamon, Oxford, UK, 1984, translated from the Russian by J. B. Sykes, J. S. Bell, and M. J. Kearsley.

[17] Z. E. Eremenko, E. M. Ganapolskii, and V. V. Vasilchenko, "Exact-calculated resonator method for permittivity measurement of high loss liquids at millimetre wavelength,"
Measurement Science and Technology, vol. 16, no. 8, pp. 16191627, 2005.

[18] Z. E. Eremenko and E. M. Ganapolskii, "Method of microwave measurement of dielectric permittivity in small volume of high loss liquid using hemispherical cavity resonator," Measurement Science and Technology, vol. 14, pp. 2096-2103, 2003.

[19] Z. E. Eremenko, "A study of the E-field dependence of resonant modes of a layered semi-ball immersed in lossy liquid," Measurement Science and Technology, vol. 18, no. 11, pp. 33033308, 2007.

[20] A. Stratton, Electromagnetic Theory, McGraw Hill, New York, NY, USA, 1941.

[21] M. Abramowitz and I. Stegun, Handbook of Mathematical Functions, National Bureau of Standards Applied Mathematics, U.S. Government Printing Office, Washington, DC, USA, 1964.

[22] S. Mashimo, S. Kuwabara, S. Yagihara, and K. Higasi, "The dielectric relaxation of mixtures of water and primary alcohol," The Journal of Chemical Physics, vol. 90, no. 6, pp. 32923294, 1989.

[23] T. Sato and R. Buchner, "Dielectric relaxation processes in ethanol/water mixtures," Journal of Physical Chemistry A, vol. 108, no. 23, pp. 5007-5015, 2004.

[24] C. L. Holloway, D. A. Hill, R. A. Dalke, and G. A. Hufford, "Radio wave propagation characteristics in lossy circular waveguides such as tunnels, mine shafts, and boreholes," IEEE Transactions on Antennas and Propagation, vol. 48, no. 9, pp. 1354-1366, 2000. 

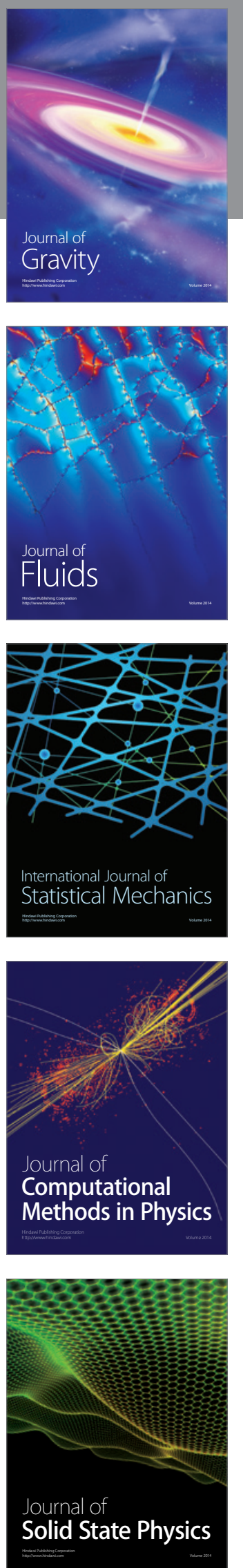

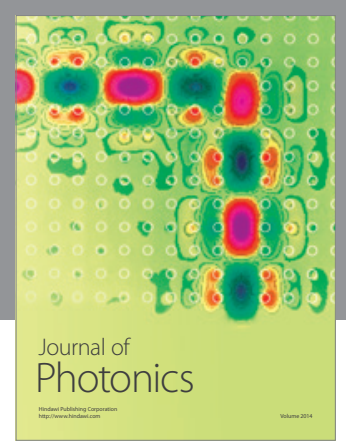

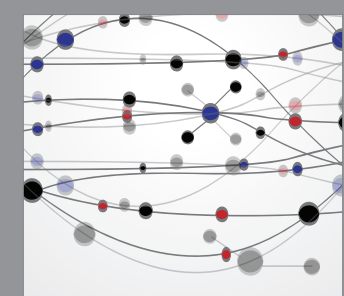

The Scientific World Journal
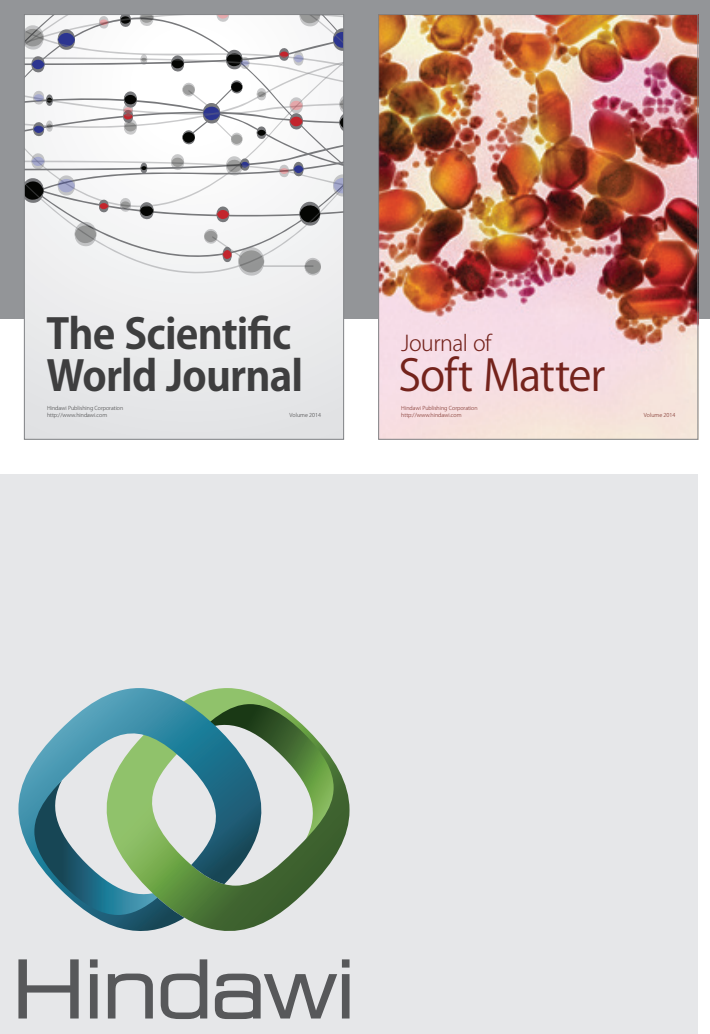

Submit your manuscripts at

http://www.hindawi.com
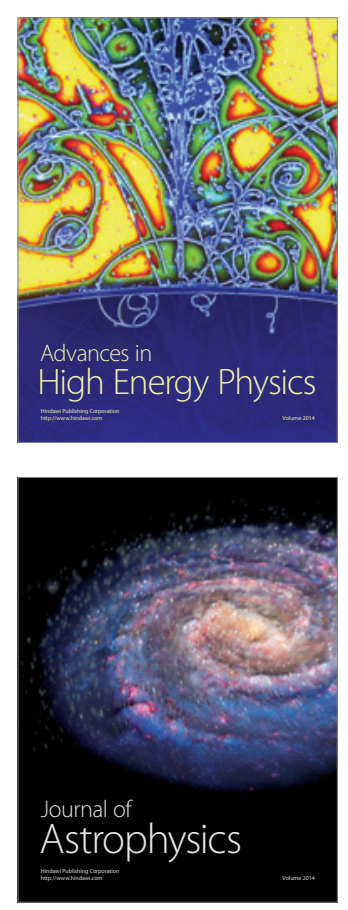
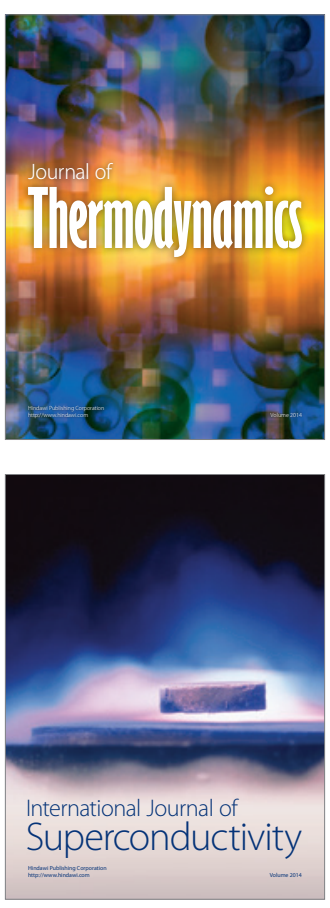
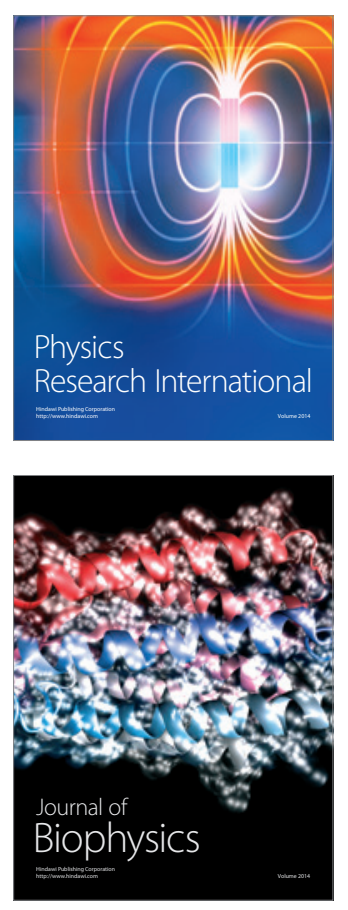
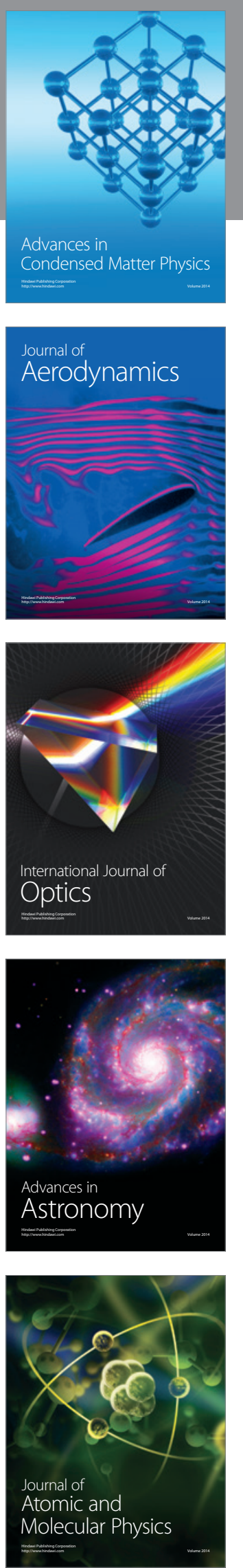